\title{
Interpersonal psychotherapy for perinatal women: a systematic review and meta- analysis protocol
}

Katherine S. Bright ${ }^{1,2^{*}}$ (D), Elyse M. Charrois ${ }^{1}$, Muhammad Kashif Mughal ${ }^{1}$, Abdul Wajid ${ }^{1}$, Deborah McNeil ${ }^{1,2,3}$, Scott Stuart ${ }^{4}$, K. Alix Hayden ${ }^{5}$ and Dawn Kingston ${ }^{1,2}$

\begin{abstract}
Background: Interpersonal psychotherapy (IPT) is an intervention that has established efficacy in the prevention and treatment of depressive disorders. Previous systematic reviews have not evaluated the effectiveness of IPT on symptoms of stress, anxiety, depression, quality of life, relationship satisfaction/quality, social supports, and an improved psychological sense of well-being. There is limited data regarding factors that moderate and mediate the effectiveness of IPT including the timing of the intervention or the mode of delivery of IPT intervention. The objective of this systematic review and meta-analysis is to evaluate the effectiveness, feasibility, and acceptability of IPT interventions to treat perinatal psychological distress and to summarize the evidence on predictors, mediators, and moderators of IPT.
\end{abstract}

Methods: We will include peer-reviewed studies that recruited perinatal women. The search strategy will involve the following databases: MEDLINE (Ovid), EMBASE (Ovid), PsycINFO (Ovid), Cochrane Central Register of Controlled Trials (Ovid), CINAHL with Full Text (EBSCO), Social Work Abstracts (EBSCO), SocINDEX with Full Text (EBSCO), Academic Search Complete (EBSCO), Family \& Society Studies Worldwide (EBSCO), Family Studies Abstracts (EBSCO), and Scopus. Study inclusion criteria include (1) randomized controlled trials, quasi-experimental studies, and prepost studies that evaluated the effectiveness of IPT; (2) qualitative studies that evaluated feasibility and acceptability of IPT; (3) study sample included and analyzed perinatal women; and (4) publication language was English. Using pilot-tested screening and data extraction forms, two reviewers will independently review studies in three steps: (1) abstract/title screening, (2) full-text screening of potentially accepted studies, and (3) data extraction of accepted studies. Disagreements will be resolved by a third reviewer. Studies will be aggregated for meta-synthesis and meta-analysis should the data allow for this. Two independent reviewers will grade methodological quality.

Discussion: Findings from this review will inform future development and implementation of IPT intervention research for perinatal women. Identifying key factors of successful IPT interventions will inform intervention design and adaptation of IPT interventions to increase the likelihood that perinatal women will engage in and benefit from IPT interventions. This review will also identify key considerations for increasing the effectiveness of IPT interventions during the perinatal period.

Systematic review registration: PROSPERO CRD42019114292

Keywords: Systematic review, Interpersonal psychotherapy, Antenatal, Perinatal, Postpartum, Women, Distress

\footnotetext{
* Correspondence: ksbright@ucalgary.ca

'Faculty of Nursing, University of Calgary, PF2226, 2500 University Drive NW,

Calgary, Alberta T2N 1N4, Canada

${ }^{2}$ Alberta Children's Hospital Research Institute (ACHRI), Calgary, Alberta,

Canada

Full list of author information is available at the end of the article
}

(c) The Author(s). 2019 Open Access This article is distributed under the terms of the Creative Commons Attribution 4.0 International License (http://creativecommons.org/licenses/by/4.0/), which permits unrestricted use, distribution, and reproduction in any medium, provided you give appropriate credit to the original author(s) and the source, provide a link to the Creative Commons license, and indicate if changes were made. The Creative Commons Public Domain Dedication waiver (http://creativecommons.org/publicdomain/zero/1.0/) applies to the data made available in this article, unless otherwise stated. 


\section{Background}

The perinatal period, from conception until 12-months postpartum, is a time of increased social, emotional, biological, and psychological adjustments [1, 2]. Additionally, the perinatal period is a developmental life stage involving significant adaptation to changes in physical appearance and expectations for new roles [3, 4]. The perinatal period is also a time of increased vulnerability to psychological stress and an impaired sense of wellbeing $[5,6]$. Epidemiological research suggests that psychological distress, including stress, anxiety, and depression, resulting from these changes occurs between 15 and $25 \%$ of perinatal women $[7,8]$. Psychological distress during the perinatal period is also associated with impaired mother-fetal/infant relationship, obstetrical complications, and child developmental problems $[9,10]$. Additionally, left untreated, up to $40 \%$ of these women will have symptoms that persist until their children enter preschool and beyond [11, 12]. However, psychological distress during the perinatal period is often under detected and untreated [13-15].

The impact of perinatal psychological distress on infants, children, women, and their families is well established. Evidence regarding the most effective psychological treatments during the perinatal period is emerging [16, 17]. There are over 35 years of research examining the use of cognitive behavior therapy (CBT) and interpersonal psychotherapy (IPT) in adult populations with the results suggesting that these treatments are equally efficacious in treating depression in the short term [18, 19] and the long term [20]. Studies examining the efficacy of IPT during the perinatal period are also promising. One pilot RCT compared the efficacy of 16 45-min individualized session in IPT (21 participants) with a parenting education program (17 participants) for new immigrants from the Dominican Republic [21]. The IPT treatment group showed significant improvements in mood compared to the parenting education control program [21]. While attrition rate in the study was high and there was greater homogeneity of the sample population both which significantly limited the generalizability of these findings, the outcome of improvements in mood in the IPT treatment group are promising [21].

An American study of group IPT for depressed pregnant women receiving public assistance found that at 3 months postpartum, no one in the IPT group had symptoms of depression compared to $33 \%$ of women in the treatment-as-usual (no therapy) group [22]. In another American study exploring group IPT in pregnant women, IPT was found to be an effective treatment for the reduction of depression symptoms in pregnancy and prevention of postpartum depression [23]. To date, studies examining IPT treatment in pregnancy are limited by their small sample sizes and thus reduced generalizability of their findings.

IPT is considered the best front-line treatment for postpartum depression (PPD) when symptoms are mild and in conjunction with antidepressant medications when symptoms are in the moderate to severe range [24-27]. In a systematic review from 2014, 11 studies summarizing the efficacy of IPT for PPD found support for both individual and group IPT interventions for women [28]. The systematic review suggested that women who engaged in IPT noted significant improvements in their marital and mother-newborn relationships. Other studies report significant improvement in depressive symptoms [29, 30].

Delivered to postpartum women, IPT focuses on increasing social supports while addressing interpersonal problems that result from a lack of help with childcare or a perceived absence of emotional support [24]. The transition into motherhood may involve decisions about new roles and focusing attention in new areas [24]. IPT during the postpartum period also may address components of grief and loss such as miscarriage, loss of independent identity, loss of employment, and closeness in intimate relationship [24].

In a recent systematic review (2018) of the efficacy of IPT in perinatal women, 28 studies endorsed the effectiveness of IPT in the prevention and/or treatment of perinatal distress [31]. The 2018 review lacked congruency with systematic literature review best practices as the search was limited to two databases, screening was completed by only one reviewer, and we were not able to replicate the search strategy results [31]. Thus, there is a need for a more systematic, comprehensive, and transparent approach to examining the use of IPT in perinatal women.

While evidence suggests that psychological therapy is effective, perinatal women report significant barriers in seeking psychological support including stigma (self and by their healthcare professional), uncertainty about whether their symptoms are normal or abnormal, inability to articulate their distress, wanting to self-manage first, not wanting to take psychotropic medications, lack of time, financial expenditure, location and proximity of services, transportation issues, and challenges associated with childcare $[8,14,32]$. As a result, instead of using formal treatments, women are more inclined to seek informal support from family, printed material, or computer/web-based intervention programs [7, 8, 33].

Individual characteristics that influence computer/ web-based treatment effectiveness can be categorized into predictors, moderators, and mediators [34]. Predictors and moderators are considered pre-treatment variables; however, the former forecasts mental health outcomes in the treatment groups, while the latter 
identifies persons more likely to benefit from which particular treatment [34]. Examples of predictors of computer/web-based interventions include when the therapy is guided by a coach/therapist, being female, and obtaining low mastery scores and dysfunctional attitudes [34, 35]. Interestingly, a moderator of internet-based therapy is age, with older adults benefiting more from CBT and younger adults noting more significant improvements with IPT [34]. For younger adults, the improvements from internet-based IPT are due to the foci of IPT; specifically, interpersonal conflicts and role transitions are particularly relevant to the stage of life for young adults [24, 34].

Additionally, IPT is an intervention aimed at alleviating psychological symptoms, coping with problems due to loss, change, and relationship conflict, thereby improving interpersonal functioning [36, 37]. IPT is a therapy based on the notion that when faced with adversity, factors such as attachment needs, communication patterns, and the quality of social support networks contribute significantly to an individual's range of symptoms of psychological distress [37]. Conceptualizations of social supports come from work on attachment theory, trust, and coping in times of adversity [38]. These social supports play an important role in how individuals manage stress and work through the coping process [39-43]. IPT attempts to improve attachment security, interpersonal change, and psychological distress [44, 45]. As a result, IPT has the potential to improve individual coping and resiliency.

\section{Objectives}

The objective of this review is to evaluate the effectiveness, feasibility, and acceptability of IPT interventions to treat perinatal psychological distress and to summarize the evidence on predictors, mediators, and moderators of IPT. The questions guiding this systematic review and meta-analysis are the following:

1. What is the effectiveness of IPT for women during the perinatal period on the reduction of stress, anxiety, depression, quality of life, relationship satisfaction/quality, social support, and improved psychological well-being?

2. What are the predictors, mediators, and moderators of IPT, including timing of IPT and mode of delivery?

3. What is the feasibility and acceptability of IPT?

\section{Methods}

\section{Protocol and registration}

The protocol for this systematic review was developed according to the Preferred Reporting Items for Systematic reviews and Meta-analyses Protocols (PRISMA-P)
[46] (see Additional file 1) and has been registered with PROSPERO CRD42019114292. We will document any amendments to the protocol with a rationale and report them with the final publication.

\section{Eligibility criteria}

The studies selected for inclusion in this systematic review will meet the following eligibility criteria which are described according to participants, study design (including publication, language, and year), intervention, and outcomes.

\section{Participants}

Perinatal women from conception to 12 months postpartum who participated in IPT intervention. For this review, women who are not pregnant or postpartum or men who engage in IPT interventions will be excluded. The rationale for this exclusion is that we are exploring the effectiveness of IPT for women during the perinatal period.

\section{Study design}

The review will consider studies evaluating the feasibility, acceptability, effectiveness, and/or efficacy of IPT in perinatal women. Experimental studies such as randomized controlled/clinical trials (RCTs), quasi-experimental studies, as well as single-group pre-post studies will be included in the review. In cases of duplicate publications of the same sample, we will include the publication with the largest sample size. If sample sizes are consistent, the first published study from the sample will be included. We will also include qualitative studies that explore the acceptability of the intervention. We will exclude conference papers, dissertations, reviews, and non-English publications.

\section{Interventions}

We define IPT intervention as an intervention, which may include interpersonal therapy, or intervention, counseling, psychotherapy, therapy, or program where there was a component of IPT offered. IPT will include those interventions targeted towards women during the perinatal period. Interventions not including IPT will be excluded.

\section{Comparator}

We will include studies with all types of comparator groups, such as non-exposed control group or a group exposed to a different intervention.

\section{Outcomes}

Primary outcome Similar to other reviews on the effectiveness of IPT for women during the perinatal 
period, this review will synthesize information on the effectiveness of IPT for a range of outcomes including stress, anxiety, depression, quality of life, relationship satisfaction/quality, social support, and improved psychological well-being. Effect sizes will be calculated based on within-group effect size and between-group effect size.

Secondary outcomes This review will also synthesize information on predictors, mediators, and moderators of IPT, including timing of IPT and mode of delivery. Predictors of treatment efficacy will include clinical or biological factors such as age and history of mental health concerns, whereas mediators of treatment efficacy measure changes that occur during treatment and these mediators correlate with treatment outcome. Examples of mediators of treatment may include interpersonal conflict and maladaptive communication skills. According to the theoretical framework, IPT works by virtue of improving conflict in relationships by improving communication skills [37]. Moderators of treatment efficacy outcomes also involves clinical or biological factors, these include the presence or magnitude of a factor at baseline that influences the relative likelihood of a particular outcome occurring following treatment with one versus another agent.

Tertiary outcomes Additional outcomes of feasibility and acceptability of IPT will be synthesized. Feasibility will consider whether women find the IPT intervention as easy to complete. Acceptability will be measured by women's perceptions of the appropriateness of the content; the intervention was found to be effective in achieving the intended outcomes.

\section{Information sources and search strategy}

MEDLINE(R) and Epub Ahead of Print, In-Process \& Other Non-Indexed Citations and Daily (Ovid); EMBASE (Ovid); PsycINFO (Ovid); Cochrane Central Register of Controlled Trials (Ovid); CINAHL with Full Text (EBSCO); Social Work Abstracts (EBSCO); SocINDEX with Full Text (EBSCO); Academic Search Complete (EBSCO); Family \& Society Studies Worldwide (EBSCO), Family Studies Abstracts (EBSCO); and Scopus databases will be searched from database inception onwards. The search strategy was developed in collaboration with an expert health sciences librarian (KAH). Keywords will be constant across databases, and subject headings will be responsive to the controlled vocabulary of each database. The MEDLINE (Ovid) search strategy is in Table 1. In addition, we will conduct citation searches in Google Scholar, search the reference lists of included studies, and ask experts to identify further studies for inclusion. No search filters or hedges will be used. Article language will be restricted to English. EndNote X8 will be used to manage the records, remove duplicates, and retrieve full-texts. Any full texts missing in Endnote will be retrieved manually. We will re-run the searches prior to the final analyses to retrieve any addition recently published articles for inclusion.

\section{Screening of studies}

Prior to screening titles and abstracts, we will conduct training and an inter-relater calibration exercise with the review team. These two reviewers (KSB and EML), who are experts in perinatal mental health, will then independently screen the remaining studies for eligibility in two steps. The first step will consist of reviewing all studies' titles/abstracts to identify studies that meet the eligibility criteria. The second step will consist of reviewing the provisionally included studies' full-text to ensure that they meet all the inclusion criteria. Any disagreements will be resolved by a third expert reviewer (MKM). The total number of studies retrieved, reviewed, included, and excluded as well as reasons for exclusion will be reported.

\section{Data items and data extraction}

Table 2 details the data items to be extracted from the studies. These items are informed by the Template for Intervention Description and Replication (TIDieR) [47] and Transparent Reporting of Evaluations with Nonrandomized Designs (TREND) checklists [48]. Extracted data will include study characteristics, participants, intervention characteristics (including length, timing, mode of delivery, and intensity), participant flow, assignment methods, recruitment methods, and retention methods. We will use a templated Microsoft Excel data extraction tool. After the team completes the inter-rater exercise with the extraction tool, one reviewer (MKM) will extract all study data with a second reviewer verifying the extracted data. Any discrepancies in extracted data will be resolved through discussion.

\section{Risk of bias in individual studies}

Studies will be included regardless of methodological quality. The Cochrane risk of bias tool [49] will be used to rate selection, performance, detection, attrition, reporting, and other biases in each study as either high, unclear, or low risk of bias. For non-randomized studies of interventions, specific to cohort studies, ROBIN-1 will be used to assess bias including overall, selection of participants, type of intervention, deviation from intended intervention, outcome measurements, and reporting of results in studies as either low risk, moderate risk, serious risk, critical risk, or no risk [50]. Two reviewers will independently assess all studies for quality, and disagreements will be resolved through a third expert if a 
Table 1 Search strategy

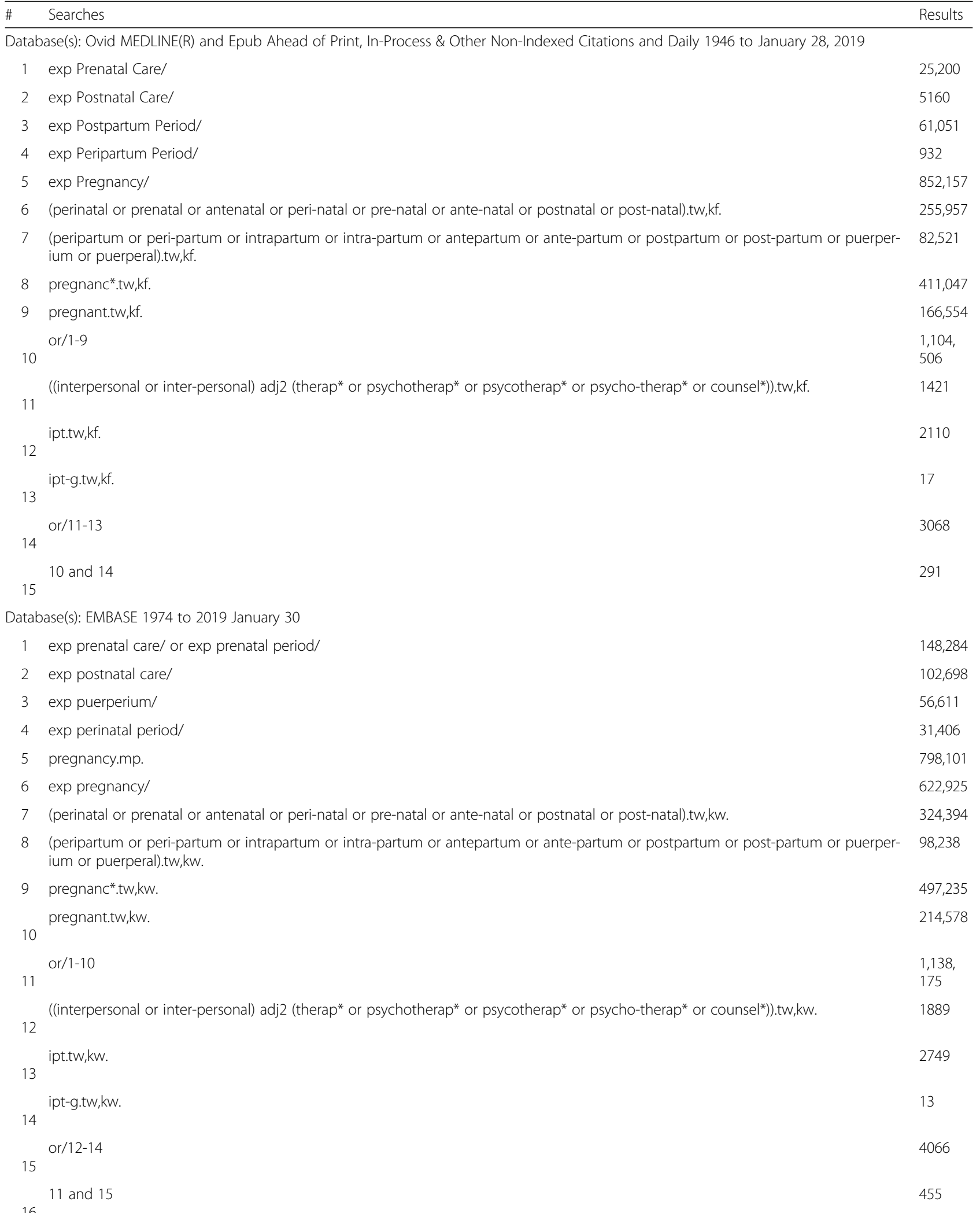

Database(s): EBM Reviews - Cochrane Central Register of Controlled Trials December 2018 
Table 1 Search strategy (Continued)

\begin{tabular}{|c|c|c|}
\hline \# & Searches & Results \\
\hline 1 & exp Prenatal Care/ & 1224 \\
\hline 2 & exp Postnatal Care/ & 352 \\
\hline 3 & exp Postpartum Period/ & 1388 \\
\hline 4 & exp Peripartum Period/ & 7 \\
\hline 5 & exp Pregnancy/ & 19,347 \\
\hline 6 & (perinatal or prenatal or antenatal or peri-natal or pre-natal or ante-natal or postnatal or post-natal).tw,kw. & 10,453 \\
\hline 7 & $\begin{array}{l}\text { (peripartum or peri-partum or intrapartum or intra-partum or antepartum or ante-partum or postpartum or post-partum or puerper- } \\
\text { ium or puerperal).tw,kw. }\end{array}$ & 7654 \\
\hline 8 & pregnanc ${ }^{*} . t w, k w$. & 26,562 \\
\hline 9 & pregnant.tw,kw. & 11,671 \\
\hline 10 & or/1-9 & 45,654 \\
\hline 11 & ((interpersonal or inter-personal) adj2 (therap* or psychotherap* or psycotherap* or psycho-therap* or counsel*)).tw,kw. & 726 \\
\hline 12 & ipt.tw,kw. & 548 \\
\hline 13 & ipt-g.tw,kw. & 8 \\
\hline 14 & or/11-13 & 970 \\
\hline 15 & 10 and 14 & 115 \\
\hline \multicolumn{3}{|c|}{ Database(s): PsycINFO 1806 to January Week 42019} \\
\hline 1 & exp PRENATAL CARE/ & 1933 \\
\hline 2 & exp POSTNATAL PERIOD/ & 4204 \\
\hline 3 & (perinatal or prenatal or antenatal or peri-natal or pre-natal or ante-natal or postnatal or post-natal).tw,id. & 43,526 \\
\hline 4 & $\begin{array}{l}\text { (peripartum or peri-partum or intrapartum or intra-partum or antepartum or ante-partum or postpartum or post-partum or puerper- } \\
\text { ium or puerperal).tw,id. }\end{array}$ & 12,895 \\
\hline 5 & pregnanc.tw,id. & 37,518 \\
\hline 6 & pregnant.tw,id. & 17,640 \\
\hline 7 & or/1-6 & 80,027 \\
\hline 8 & exp Interpersonal Psychotherapy/ & 1283 \\
\hline 9 & ((interpersonal or inter-personal) adj2 (therap* or psychotherap* or psycotherap* or psycho-therap* or counsel*)).tw,id. & 3014 \\
\hline 10 & ipt.tw,id. & 1094 \\
\hline 11 & ipt-g.tw,id. & 24 \\
\hline 12 & or/8-11 & 3536 \\
\hline 13 & 7 and 12 & 156 \\
\hline
\end{tabular}

disagreement cannot be solved among the two initial reviewers.

\section{Data synthesis}

Synthesis of the data will be conducted according to the Cochrane guidance [51]. Should the data permit, a metaanalysis will be conducted using Comprehensive MetaAnalysis (CMA) software, version 3.0. Additionally, should data permit, a meta-synthesis will be conducted on the qualitative studies.

Recruitment and retention will be calculated as a proportional effect size with confidence intervals reported based on a 95\% criterion. These proportions will be computed first as a proportional effect size and then transformed into logit units using CMA, which allows for more variability in the data than the bound 
Table 2 Data extraction categories for eligible studies

\begin{tabular}{|c|c|}
\hline Category & Data extracted \\
\hline $\begin{array}{l}\text { Study } \\
\text { characteristics }\end{array}$ & First author, year, and country \\
\hline Participant & $\begin{array}{l}\text { Eligibility criteria (including different levels in } \\
\text { recruitment/sampling plan), demographics, and } \\
\text { data collection setting }\end{array}$ \\
\hline $\begin{array}{l}\text { Intervention } \\
\text { characteristics }\end{array}$ & $\begin{array}{l}\text { Content, delivery method, unit of delivery, deliverer, } \\
\text { setting, exposure quantity and duration, and time } \\
\text { span }\end{array}$ \\
\hline \multirow{3}{*}{$\begin{array}{l}\text { Assignment } \\
\text { method }\end{array}$} & Unit of assignment \\
\hline & $\begin{array}{l}\text { Method used to assign units to study conditions, } \\
\text { including details of any restriction }\end{array}$ \\
\hline & $\begin{array}{l}\text { Inclusion of aspects employed to help minimize } \\
\text { potential bias comparison condition induced due } \\
\text { to nonrandomization }\end{array}$ \\
\hline Participant flow & $\begin{array}{l}\text { Flow of participants: enrollment, assignment, } \\
\text { allocation and intervention exposure, baseline and } \\
\text { outcome measures, and follow-up }\end{array}$ \\
\hline Recruitment & $\begin{array}{l}\text { Method of recruitment, sampling method, and } \\
\text { recruitment setting }\end{array}$ \\
\hline Retention & Method to increase compliance or adherence \\
\hline Outcomes & $\begin{array}{l}\text { Stress, anxiety, depression, quality of life, } \\
\text { relationship satisfaction/quality, social support, and } \\
\text { improved psychological well-being }\end{array}$ \\
\hline
\end{tabular}

proportional effect sizes [52, 53]. Recruitment rates will be calculated using previously described approaches [54-56] that calculate a rate based on the number of participants who started participation at each recruitment site divided by the total recruitment period (defined as the time in months between the start and completion of recruitment). We will calculate retention based on a previously described strategy [57], in which the number of participants (perinatal women) retained in perinatal IPT research until the time point of primary assessment outcome was collected is compared to the total number of participants enrolled. To calculate attrition rates, we will calculate the number of perinatal women lost during follow-up out of duration between follow-up time points. Feasibility will also consider whether women find the IPT intervention as easy to complete. Acceptability will be measured by women's perceptions of the appropriateness of the content, found the intervention effective in achieving the intended outcomes.

We will also conduct a sensitivity analysis by sequentially removing one study at a time and reanalyzing the dataset to determine the impact of any given single study. In conjunction with the quality analysis previously mentioned, this procedure allows for the inclusion of methodologically flawed studies if they meet this criterion. Provided that there is enough information, we will also carry out meta-regression using logit units to explore potential modifiers of recruitment and retention rates, such as (a) study characteristics, (b) recruitment methods, (c) retention methods, (d) maternal and family characteristics, and (e) intervention characteristics. In the case that the included studies are too heterogeneous for meta-analysis, we will aggregate findings using narrative synthesis [58].

\section{Missing data}

Authors of studies will be contacted to obtain missing data.

\section{Assessment of heterogeneity}

Heterogeneity of the studies' recruitment and retention proportions will be assessed by the non-parametric Cochrane $Q$ test, which assesses variance between studies and study populations. The $I^{2}$ index will be calculated to evaluate the proportion of heterogeneity between studies. If heterogeneity is present, random-effect models (as opposed to fixed-effect models) will be used as they are a more appropriate computational approach under conditions of heterogeneity given they are less likely to reject the null hypothesis. Further, randomeffect models are more robust to large variations in sample sizes [59].

\section{Subgroup analysis}

Data permitting, subgroup analysis will be carried out considering participant characteristics (such as stage in pregnancy/postpartum, maternal age, previous children, previous losses) and intervention characteristics (when it was delivered, mode of delivery, dose, frequency, and duration).

\section{Meta-analysis of bias}

Publication bias and selection of variables in publications will be assessed through visual inspection of a funnel plot as well as statistical tests (e.g., Egger's regression intercept, Begg and Mazumdar's rank correlation, and Orwin's fail-safe $N$ ) [60-62].

\section{Discussion}

Findings from this review will inform future development and implementation of IPT intervention research for perinatal women. Identifying key factors of successful IPT interventions will enable the design and adaptation of IPT interventions to increase the likelihood of perinatal women engaging in and benefiting from IPT interventions during the perinatal period. Researchers will be able to use this review to inform future research that addresses current evidence gaps of IPT interventions for women during pregnancy and 1 year postpartum. This review will also identify key mediators and moderators of IPT interventions to enhance design, implementation, and uptake of IPT. 
Limitations to the review may include limited data that could impede the ability to run meta-analyses on all potential sub-groups of participant and study characteristics. Additionally, there may be limited descriptions of recruitment and retention strategies as well as timing of the intervention in the articles under review. Further limitations are related to the inclusion/exclusion criteria of reviewing only English language articles, which may reduce generalizability to non-English-speaking populations. Similarly, the inclusion of only peer-reviewed literature excludes government reports, dissertations, conference papers, and reviews thereby potentially limiting grass-roots or community-based recruitment and retention strategies that may be used to target smaller or marginalized groups of perinatal women.

\section{Supplementary information}

Supplementary information accompanies this paper at https://doi.org/10. 1186/s13643-019-1158-6

Additional file 1: PRISMA-P 2015 Checklist.

\section{Abbreviations}

PRISMA-P: Preferred Reporting Items for Systematic reviews and Metaanalyses Protocols; TIDieR: Template for Intervention Description and Replication; TREND: Transparent Reporting of Evaluations with Nonrandomized Designs

\section{Acknowledgements}

The authors are grateful for the support of the University of Calgary, Faculty of Nursing, and the Alberta Children's Hospital Research Institute.

\section{Authors' contributions}

KSB and DK conceived the review. All authors designed the protocol. AK and KSB conducted the preliminary searches. All authors reviewed the manuscript, read, and approved the final manuscript. DK is the guarantor of the review.

\section{Funding}

KSB is supported by the Graduate Studentship Award from the ACHRI and Faculty of Nursing, University of Calgary. EML is supported by the Eyes High Doctoral Scholarship, University of Calgary.

\section{Availability of data and materials}

The datasets created and analyzed during this review will be available from the corresponding author upon reasonable request. We will also deposit our datasets in an open access repository, Open Science Framework (https://osf. io/), developed and maintained by the Centre for Open Science.

\section{Ethics approval and consent to participate}

Not applicable

\section{Consent for publication}

Not applicable

\section{Competing interests}

The authors declare that they have no competing interests.

\section{Author details}

${ }^{1}$ Faculty of Nursing, University of Calgary, PF2226, 2500 University Drive NW, Calgary, Alberta T2N 1N4, Canada. ${ }^{2}$ Alberta Children's Hospital Research Institute (ACHRI), Calgary, Alberta, Canada. ${ }^{3}$ Alberta Health Services, Southport Atrium \#2237, 10101 Southport Road, S.W., Calgary, Alberta, Canada. ${ }^{4}$ Psychiatry/Psychological \& Brain Sciences, University of lowa, 1-293 MEB, W311 Seashore Hall, lowa City, IA 55241-1407, USA. ${ }^{5}$ Libraries and
Cultural Resources, University of Calgary, 2500 University Dr. N.W., Calgary, Alberta T2N 1N4, Canada.

Received: 23 May 2019 Accepted: 13 September 2019

Published online: 29 October 2019

\section{References}

1. Huizink A, Menting B, De Moor M, Verhage M, Kunseler F, Schuengel C, et al. From prenatal anxiety to parenting stress: a longitudinal study. Arch Womens Ment Health. 2017;20(5):663-72.

2. Ashford MT, Olander EK, Ayers S. Computer-or web-based interventions for perinatal mental health: a systematic review. J Affect Disord. 2016;197:134-46.

3. Göbel A, Stuhrmann LY, Harder S, Schulte-Markwort M, Mudra S. The association between maternal-fetal bonding and prenatal anxiety: an explanatory analysis and systematic review. J Affect Disord. 2018;239:313-27.

4. Smith MV, Shao L, Howell H, Lin H, Yonkers KA. Perinatal depression and birth outcomes in a Healthy Start project. Matern Child Health J. 2011;15(3):401-9.

5. Biaggi A, Conroy S, Pawlby S, Pariante CM. Identifying the women at risk of antenatal anxiety and depression: a systematic review. J Affect Disord. 2016; 191:62-77.

6. Tomfohr LM, Buliga E, Letourneau NL, Campbell TS, Giesbrecht GF. Trajectories of sleep quality and associations with mood during the perinatal period. Sleep. 2015;38(8):1237-45.

7. Kingston D, Austin M-P, Hegadoren K, McDonald S, Lasiuk G, McDonald S, et al. Study protocol for a randomized, controlled, superiority trial comparing the clinical and cost-effectiveness of integrated online mental health assessment-referral-care in pregnancy to usual prenatal care on prenatal and postnatal mental health and infant health and development: the Integrated Maternal Psychosocial Assessment to Care Trial (IMPACT). Trials. 2014;15(1):72.

8. Loughnan SA, Newby JM, Haskelberg H, Mahoney A, Kladnitski N, Smith J, et al. Internet-based cognitive behavioural therapy (iCBT) for perinatal anxiety and depression versus treatment as usual: study protocol for two randomised controlled trials. Trials. 2018;19(1):56

9. Stein A, Pearson RM, Goodman SH, Rapa E, Rahman A, McCallum M, et al. Effects of perinatal mental disorders on the fetus and child. Lancet. 2014; 384(9956):1800-19.

10. Glasheen C, Richardson GA, Fabio A. A systematic review of the effects of postnatal maternal anxiety on children. Arch Womens Ment Health. 2010; 13(1):61-74

11. Horwitz SM, Briggs-Gowan MJ, Storfer-Isser A, Carter AS. Persistence of maternal depressive symptoms throughout the early years of childhood. J Women's Health (Larchmt). 2009;18(5):637-45.

12. Giallo R, Bahreinian S, Brown S, Cooklin A, Kingston D, Kozyrskyj A. Maternal depressive symptoms across early childhood and asthma in school children: findings from a longitudinal Australian population based study. PLoS One. 2015;10(3):e0121459.

13. Austin M-P, Kingston D. Psychosocial assessment and depression screening in the perinatal period: benefits, challenges and implementation. In: Joint care of parents and infants in perinatal psychiatry: Cham: Springer; 2016. p. 167-95.

14. Woolhouse H, Brown S, Krastev A, Perlen S, Gunn J. Seeking help for anxiety and depression after childbirth: results of the Maternal Health Study. ArchWomens Ment Health. 2009:12(2):75-83.

15. Austin MP, Frilingos M, Lumley J, Hadzi-Pavlovic D, Roncolato W, Acland S, et al. Brief antenatal cognitive behaviour therapy group intervention for the prevention of postnatal depression and anxiety: a randomised controlled trial. J Affect Disord. 2008;105(1-3):35-44.

16. Dennis $\mathrm{CL}$, Hodnett ED. Psychosocial and psychological interventions for treating postpartum depression. Cochrane Database Syst Rev. 2007:(4).

17. Dimidjian S, Goodman SH. Preferences and attitudes toward approaches to depression relapse/recurrence prevention among pregnant women. Behav Res Ther 2014:54:7-11.

18. Donker T, Bennett $K$, Bennett A, Mackinnon A, van Straten A, Cuijpers $P$ et al. Internet-delivered interpersonal psychotherapy versus internetdelivered cognitive behavioral therapy for adults with depressive symptoms: randomized controlled noninferiority trial. J Med Internet Res. 2013;15(5):e82

19. Westen D, Morrison K. A multidimensional meta-analysis of treatments for depression, panic, and generalized anxiety disorder: an empirical 
examination of the status of empirically supported therapies: American Psychological Association; 2001.

20. Lemmens LH, van Bronswijk SC, Peeters F, Arntz A, Hollon SD, Huibers MJ. Long-term outcomes of acute treatment with cognitive therapy $\mathrm{V}$. interpersonal psychotherapy for adult depression: follow-up of a randomized controlled trial. Psychol Med. 2019;49(3):465-73.

21. Spinelli MG, Endicott J. Controlled clinical trial of interpersonal psychotherapy versus parenting education program for depressed pregnant women. Am J Psychiatry. 2003;160(3):555-62.

22. Zlotnick C, Johnson SL, Miller IW, Pearlstein T, Howard M. Postpartum depression in women receiving public assistance: pilot study of an interpersonal-therapy-oriented group intervention. Am J Psychiatry. 2001; 158(4):638-40.

23. Spinelli MG. Interpersonal psychotherapy for depressed antepartum women: a pilot study. Am J Psychiatry. 1997;154(7):1028-30.

24. Stuart S. Interpersonal psychotherapy for postpartum depression. Clin Psychol Psychother. 2012;19(2):134-40.

25. O'Hara MW, Stuart S, Gorman LL, Wenzel A. Efficacy of interpersonal psychotherapy for postpartum depression. Arch Gen Psychiatry. 2000;57(11): 1039-45.

26. Stuart S, Clark E. The treatment of postpartum depression with interpersonal psychotherapy and interpersonal counseling. Sante Ment Que. 2008;33(2): 87-104.

27. Association AP. Clinical practice guideline for the treatment of depression across three age cohorts: American Psychological Association, Guideline Development Panel for the Treatment of Depressive Disorders; 2019.

28. Miniati M, Callari A, Calugi S, Rucci P, Savino M, Mauri M, et al. Interpersonal psychotherapy for postpartum depression: a systematic review. Arch Womens Ment Health. 2014;17(4):257-68.

29. Sockol LE, Epperson CN, Barber JP. A meta-analysis of treatments for perinatal depression. Clin Psychol Rev. 2011;31(5):839-49.

30. Grote NK, Bledsoe SE, Swartz HA, Frank E. Feasibility of providing culturally relevant, brief interpersonal psychotherapy for antenatal depression in an obstetrics clinic: a pilot study. Res Soc Work Pract. 2004;14(6):397-407.

31. Sockol LE. A systematic review and meta-analysis of interpersonal psychotherapy for perinatal women. J Affect Disord. 2018;232:316-28.

32. Kingston D, Austin M-P, Heaman M, McDonald S, Lasiuk G, Sword W, et al. Barriers and facilitators of mental health screening in pregnancy. J Affect Disord. 2015;186:350-7.

33. Griffiths KM, Christensen H. Internet-based mental health programs: a powerful tool in the rural medical kit. Aust J Rural Health. 2007;15(2):81-7.

34. Donker T, Batterham P, Warmerdam L, Bennett K, Bennett A, Cuijpers P, et al. Predictors and moderators of response to internet-delivered interpersonal psychotherapy and cognitive behavior therapy for depression. J Affect Disord. 2013:151(1):343-51.

35. Heber E, Ebert D, Lehr D, Cuijpers P, Berking M, Nobis $S$, et al. The benefit of web-and computer-based interventions for stress: a systematic review and meta-analysis. J Med Internet Res. 2017;19(2):e32.

36. Stuart S. What is IPT? The basic principles and the inevitability of change. J Contemp Psychother. 2008;38(1):1-10.

37. Stuart S, Robertson M. Interpersonal psychotherapy $2 \mathrm{E}$ a clinician's guide: CRC Press; 2012

38. Cutrona CE, Russell DW. Autonomy promotion, responsiveness, and emotion regulation promote effective social support in times of stress. Curr Opin Psychol. 2017;13:126-30.

39. Schwarzer R, Knoll N. Functional roles of social support within the stress and coping process: a theoretical and empirical overview. Int J Psychol. 2007; 42(4):243-52.

40. Nurullah AS. Received and provided social support: a review of current evidence and future directions: 2012.

41. Champion L. Social relationships and social roles. Clin Psychol Psychother. 2012:19(2):113-23.

42. Cohen S, McKay G. Social support, stress and the buffering hypothesis: a theoretical analysis. Handb Psychol Health. 1984:4:253-67.

43. Cohen S, Wills TA. Stress, social support, and the buffering hypothesis Psychol Bull. 1985:98(2):310.

44. Grigoriadis S, Ravitz P. An approach to interpersonal psychotherapy for postpartum depression focusing on interpersonal changes. Can Fam Physician. 2007;53(9):1469-75.

45. Koszycki D, Bisserbe J-C, Blier P, Bradwejn J, Markowitz J. Interpersonal psychotherapy versus brief supportive therapy for depressed infertile women: first pilot randomized controlled trial. Arch Womens Ment Health. 2012:15(3):193-201.

46. Moher D, Shamseer L, Clarke M, Ghersi D, Liberati A, Petticrew M, et al. Preferred reporting items for systematic review and meta-analysis protocols (PRISMA-P) 2015 statement. Syst Rev. 2015;4(1):1.

47. Hoffmann TC, Glasziou PP, Boutron I, Milne R, Perera R, Moher D, et al. Better reporting of interventions: template for intervention description and replication (TIDieR) checklist and guide. BMJ (Clinical research ed). 2014;348: g1687.

48. Des Jarlais DC, Lyles C, Crepaz N, Group T. Improving the reporting quality of nonrandomized evaluations of behavioral and public health interventions: the TREND statement. Am J Public Health. 2004;94(3):361-6.

49. Higgins JP, Altman DG, Gotzsche PC, Juni P, Moher D, Oxman AD, et al. The Cochrane collaboration's tool for assessing risk of bias in randomised trials. BMJ (Clinical research ed). 2011;343:d5928.

50. Sterne J, Higgins J, RG E RB. Risk of bias in non-randomised studies of interventions (ROBINS-I): detailed guidance, updated 12 October 2016. 2016.

51. Higgins J, Green S, editors. Cochrane handbook for systematic reviews of interventions. Version 5.1.0 [updated March 2011]. The Cochrane Collaboration. Available from http://handbook.cochrane.org/; 2011.

52. Lipsey M, Wilson D. Practical meta-analysis. Thousand Oaks: Sage; 2001.

53. Snijders TAB, Bosker RJ. Multilevel analysis: an introduction to basic and advanced multilevel modelling. 2nd ed. London: Sage; 2012.

54. Walters SJ, Bonacho dos Anjos Henriques-Cadby I, Bortolami O, Flight L, Hind D, Jacques RM, et al. Recruitment and retention of participants in randomised controlled trials: a review of trials funded and published by the United Kingdom Health Technology Assessment Programme. BMJ Open. 2017;7(3):e015276.

55. Cooke R, Jones A. Recruiting adult participants to physical activity intervention studies using sport: a systematic review. BMJ Open Sport Exerc Med. 2017:3(1):e000231.

56. Foster CE, Brennan G, Matthews A, McAdam C, Fitzsimons C, Mutrie N. Recruiting participants to walking intervention studies: a systematic review. Int J Behav Nutr Phys Act. 2011;8:137.

57. Brueton VC, Tierney JF, Stenning S, Meredith S, Harding S, Nazareth I, et al. Strategies to improve retention in randomised trials: a Cochrane systematic review and meta-analysis. BMJ Open. 2014:4(2):e003821.

58. Popay J, Roberts H, Sowden A, Petticrew M, Arai L, Rodgers M, et al. Guidance on the conduct of narrative synthesis in systematic reviews. Lancaster: ESRC Research Methods Programme; 2006.

59. Cooper H, Hedges LV, Valentine JC, editors. The handbook of research synthesis and meta-analysis. New York: Russell Sage Foundation; 2009.

60. Begg CB, Mazumdar M. Operating characteristics of a rank correlation test for publication bias. Biometrics. 1994;50(4):1088-101.

61. Egger M, Smith GD, Schneider M, Minder C. Bias in meta-analysis detected by a simple, graphical test. BMJ. 1997;315(7109):629-34.

62. Orwin RG. A fail-safe N for effect size in meta-analysis. J Educ Stat. 1983;8(2): $157-9$

\section{Publisher's Note}

Springer Nature remains neutral with regard to jurisdictional claims in published maps and institutional affiliations.

Ready to submit your research? Choose BMC and benefit from:

- fast, convenient online submission

- thorough peer review by experienced researchers in your field

- rapid publication on acceptance

- support for research data, including large and complex data types

- gold Open Access which fosters wider collaboration and increased citations

- maximum visibility for your research: over $100 \mathrm{M}$ website views per year

At BMC, research is always in progress.

Learn more biomedcentral.com/submission 